\title{
LA INCIDENCIA ECONÓMICA DE LAS SALVAGUARDIAS EN EL ECUADOR EN EL SECTOR DE LA ALIMENTACIÓN PERÍODO 2014 - 2016
}

\author{
THE ECONOMIC IMPACT OF SAFEGUARDS IN ECUADOR IN THE FOOD \\ SECTOR PERIOD 2014 - 2016
}

\author{
Luis Barreno Benavides \\ Universidad Tecnológica Equinoccial \\ lbarreno@ute.edu.ec
}

RECIBIDO: 03 / 04 / 2017

APROBADO: 19 / 05 / 2017

\begin{abstract}
Resumen
El objetivo del presente artículo es indicar la incidencia económica en el sector alimenticio por efecto de las salvaguardias aplicadas a las importaciones de alimentos en el Gobierno ecuatoriano durante el período 2014 - 2016. Se han analizado temas relacionados como comercio exterior, aranceles, salvaguardias, por ser el sector de la alimentación el afectado se abordó la seguridad alimentaria, los tipos de productos alimenticios que forman parte de la oferta alimentaria de un país, así como el entorno y aplicación de medidas políticas y su impacto económico. Los resultados obtenidos permitieron demostrar que en el sector de alimentos, las importaciones en las partidas afectadas por las salvaguardias ascendió a 564.6 millones de dólares, de los cuales la incidencia directa en la economía de la sociedad ecuatoriana fue un incremento en el pago de 142,4 millones de dólares por efecto de la aplicación de las salvaguardias en los productos alimenticios importados, lo que afectó directamente a la economía de los ecuatorianos y a la disminución del nivel de vida por la reducción de la oferta alimentaria del país.
\end{abstract}

Palabras clave: importaciones, aranceles, salvaguardia, seguridad alimentaria

\begin{abstract}
The objective of this article is to indicate the economic impact in the food sector due to the effect of safeguards applied to food imports in the Ecuadorian Government during the period 2014 - 2016. Related issues have been analyzed such as foreign trade, tariffs, safeguards, as the food sector affected the food security, the types of food products that are part of the food supply of a country, as well as such as the environment and implementation of policy measures and their economic impact.The results showed that in the food sector, the imports in the safeguards affected items amounted to 564.6 million dollars, of which the direct impact on the economy of the Ecuadorian society was an increase in the payment of US \$ 142.4 million due to the application of safeguards on imported food products, which directly affected the economy of Ecuadorians and reduced living standards due to the reduction of the country's food supply.
\end{abstract}

Key words: imports, tariffs, safeguard, food security

\section{Introducción}

La mayor parte de la política económica de carácter tributario adoptada por los gobiernos de turno, han perseguido un objetivo, incrementar la recaudación tributaria, para mejorar los ingresos del presupuesto general del estado, y así cubrir el déficit o desbalance ocasionados por políticas económicas internas o externas; para el caso ecuatoriano ambas razones fueron aplicadas. En cuanto a las inversiones programadas y ejecutadas por el estado para obras del sector público rebasaron la capacidad del presupuesto y además el precio internacional del petróleo, una de las principales fuentes de ingresos, se redujo considerablemente, lo que provocó un desbalance en el ingreso del presupuesto, a estas dos razones, acompañó la apreciación del dólar en Colombia y Perú, convirtiéndose en mercados atractivos para las importaciones legales, así como para el contrabando de mercancías de los ecuatorianos, 
afectando en forma significativa la balanza comercial que ya de por si era negativa especialmente con Colombia.

Con esta justificación el gobierno de Ecuador, tomó la decisión de incrementar los aranceles a unos 2800 productos importados de todo el mundo, con el fin de recuperar los ingresos perdidos, se incluyeron numerosos productos alimenticios de consumo final y materias primas para producir bienes finales, con el consiguiente incremento de los precios, lo que derivó en la disminución de la demanda, todo ello en un país en donde existen elevados niveles de desnutrición, especialmente en los hogares de escasos recursos.

Antes de iniciar con el análisis de las salvaguardias, es preciso realizar un breve repaso de algunos términos técnicos.

\section{Comercio exterior}

El comercio exterior se concibe como un conjunto de operaciones de importación y de exportación de bienes y de servicios, caracterizadas por una fuerte presencia pública en cuanto que realizadas por un Estado determinado y reguladas por su derecho administrativo económico o por el Derecho comunitario, en el caso de países pertenecientes a la Unión Europea o por otro Derecho suprarregional similar. (Rodriguez, 2012)

En esta definición se analiza al comercio exterior desde la perspectiva del derecho que los países tienen de exportar y de importar, cumpliendo con las normas establecidas en los tratados y convenios internacionales, sin embargo este derecho es afectado, cuando los gobiernos de turno con o sin razón imponen en forma unilateral obstáculos para ello, con lo que se restringe la voluntad de realizar transacciones comerciales y con eso se afecta a varios sectores incluso aquellos vulnerables como el de alimentos.

\section{Importaciones}

Es un régimen aduanero que permite el ingreso legal de mercancías provenientes del exterior para ser destinadas al consumo, las que previo cumplimiento de todas las formalidades aduaneras correspondientes, son nacionalizadas y quedan a libre disposición del dueño o consignatario (Rodriguez, 2012). Desde esta perspectiva, las importaciones de productos para el consumo tanto de bienes finales o intermedios, en un país en donde existe un déficit de alimentos y por consiguiente importantes porcentajes de desnutrición, debería ser prioritarios y deben estar exentos de impuestos, y solo puede tener restricciones cuando la producción nacional pueda cubrir en forma total la demanda de los mismos.

\section{Política arancelaria}

Desde la concepción de David Ricardo, con su teoría de las ventajas comparativas, se advirtió en forma general que el comercio mundial tenía que dejar ganancias o beneficios a todos sus participantes, sin embargo, en la práctica no es así, debido a que casi todos los países y más aún aquellos en vía al desarrollo suelen proteger la producción nacional, entre otras razones para aprovechar las ventajas comparativas de cada país, caso ecuatoriano la ubicación del país permite producir los 365 días del año, sumando además la calidad del producto primario.

El instrumento que los gobiernos utilizan para esta protección son los aranceles, a través de un gravamen denominado ad valoren, estos se aplican utilizando porcentajes más elevados que los regulares según los bienes que se quiera proteger, colocando un impuesto adicional a las bienes o mercancías importadas sobre el acordado en los tratados de comercio establecidos por los organismos de control mundial como es la Organización Mundial del Comercio (OMC), estos gravámenes se conoce normalmente como aranceles (Juan Gongora, 2010), mismos que distorsionan los resultados del comercio, tanto en la distribución de las ganancias como en los ganadores y perdedores de las mismas. 
Pero en el caso del Ecuador, el objetivo de colocar o imponer un arancel más alto del estipulado en los acuerdos comerciales internacionales, no fue exclusivamente el de proteger a la industria nacional o el de corregir la balanza de pagos, sino más bien el de incrementar las recaudaciones tributarias mediante la recaudación de ese tributo para cubrir el déficit presupuestario de ingresos dejado por la baja del precio del petróleo y la apreciación del dólar en los países vecinos como ya se manifestó anteriormente. Sin embargo, en forma indirecta, esta medida permitió disminuir en algo el déficit de la balanza comercial al reducir las cantidades importadas por el efecto de esta medida, pero por otro lado la afectación fue mayor al encarecer los bienes importados en el sector de alimentos.

\section{Las salvaguardias}

Existen diversas definiciones acerca de una salvaguardia, así (Saldaña, 2015) en términos generales, señala que se pueden definir como aquellas medidas temporales de restricción no arancelaria y de carácter cuantitativo, excepción al principio de arancel, única medida de protección a la industria, que permite a un país importador miembro de la Organización Mundial de Comercio, en casos de emergencia, limitar o restringir las importaciones de una mercancía, cualquiera que sea su origen (salvaguardia global) o solo de uno o varios países (salvaguardia bilateral), en los casos en que a consecuencia de la evolución imprevista de la circunstancias y por efecto del cumplimiento de obligaciones contraídas por una parte contratante, incluida la desgravación arancelaria, las importaciones de dicha mercancía hubiesen aumentado en tal cantidad y en condiciones tales que causen o amenacen ocasionar un daño grave a la rama de producción nacional de mercancías similares o directamente competidoras.

La Organización Mundial de Comercio, la define como medidas de urgencia con respecto al aumento de las importaciones de determinados productos cuando esas importaciones hayan causado o amenacen causar un daño grave a la rama de producción nacional del miembro importador ((OMC), 1994)

Analizado las definiciones de salvaguardias, estas son coincidentes ya que constituyen medidas de urgencia que los distintos países pueden adoptar solo en caso de que las importaciones hayan causado daños graves al país miembro. En el caso de Ecuador, si bien los resultados de la balanza comercial en forma general presentan datos negativos, sin embargo, con algunos mercados regionales inclusive se mantienen balanzas comerciales positivas por ejemplo con la Unión Europea.

Una política restrictiva o de protección para la industria nacional puede ser beneficiosa siempre y cuando internamente exista o vaya acompañada de políticas complementarias, como son líneas de crédito para la adquisición de maquinarias y equipos, adquisición de tecnología, materias primas, materiales directos e indirectos, que permitan incentivar a la industria nacional a reemplazar o sustituir las importaciones sin elevar el precio de los bienes, ofertando productos de calidad que satisfagan las necesidades de los consumidores, pero eso no ocurrió en Ecuador, el efecto fue el incremento de los precios de los bienes importados afectados por la sobretasa arancelaria, perjudicando directamente a los consumidores de bienes finales, e intermedios, por cuanto los dueños de las industrias que importan bienes de consumo intermedio para producir bienes de consumo final solo transfirieron los incrementos de los costos al consumidor final.

La salvaguardia aplicada por el gobierno ecuatoriano corresponde a una de tipo global, que según (Saldaña, 2015), tienen un efecto denominado "escopeta", ya que se impone a una mercancía, cualquiera que sea su origen, salvo las establecidas en el acuerdo sobre salvaguardias (AS) y en los tratados bilaterales que contengan disposiciones sobre el particular. Esta se diferencia del tipo bilateral, por cuanto esta última se aplica únicamente con los países que se tiene firmado tratados o acuerdos comerciales. Como se conoce las salvaguardias que el Ecuador aplicó fueron para 2800 partidas arancelarias sin importar el país de origen, por lo tanto se trata de la salvaguardia global.

Hay que señalar, que si bien se dijo que antes de gravar con la sobretasa a las partidas arancelarias de producto, primeramente se realizó un análisis pormenorizado de todas y cada 
una de ellas, sin embargo, se incluyeron partidas que contenían materias primas y especialmente las semillas para el sector agrícola.

\section{Seguridad Alimentaria}

Héctor Maletta en el año 2012 citando en el documento de las cumbres presidenciales de Centro América del año 2002, dice que la seguridad alimentaria y nutricional es un estado en el cual todas las personas gozan, en forma oportuna y permanente, de acceso físico, económico y social a los alimentos que necesitan, en cantidad y calidad, para su adecuado consumo y utilización biológica, garantizándoles un estado de bienestar general que coadyuve al logro del desarrollo (Maletta, 2011).

Esta definición de seguridad alimentaria y nutricional expresa claramente que ésta existe siempre y cuando haya disponibilidad suficiente en cantidad, calidad para que la población pueda acceder a los alimentos sin restricción alguna, esto significa que deben coexistir dos elementos al mismo tiempo, lo primero la oferta en cantidad y calidad suficientes de bienes alimenticios y la segunda la posibilidad de tener acceso a los mismos. En la política adoptada por el gobierno ecuatoriano a partir del 2014 que continuó en el año 2015, 2016 y el primer semestre del 2017, se afectaron a estos dos puntos sensibles para los consumidores, por cuanto al elevarse la tasa arancelaria ad-valoren, aun manteniéndose las cantidades ofertadas, los precios de los bienes importados de carácter alimenticio se elevaron, trayendo consigo la imposibilidad económica para adquirir dichos productos.

Otra causa que afectó a la población como consecuencia de la medida de las salvaguardias, constituye la disminución de las cantidades importadas, debido al impacto sicológico de los empresarios debido a la teoría de las elasticidades, esto es al subir el precio del producto, la demanda tiende a disminuir en esa misma proporción, el efecto de estas causas se demostrará más adelante en el análisis de los resultados estadísticos, esto contribuyó en forma directa a la disminución de la oferta alimentaria en Ecuador.

\section{Bienes o productos alimenticios}

Los bienes o productos alimenticios afectados son de dos tipos, los primeros denominados como bienes y servicios públicos de primera necesidad, o llamados también como bienes y servicios mínimos para una vida considerada socialmente digna que debe poseer cualquier persona desde que nace hasta que muere (Parramon, 2015). En lo esencial, son precisamente en ese tipo de bienes de carácter alimenticio, en su mayoría de primera necesidad en los que se ha procedido a aplicar la sobretasa arancelaria.

Dentro de los bienes de primera necesidad se encuentran los de consumo final, estos según (Jimenez, 2015), son aquellos que satisfacen directamente las necesidades, son el resultado final de la producción y la fabricación. Son los que el consumidor encuentre en la tienda y compre, por ejemplo la comida, la bebida, dentro de los alimenticios, son mercancías que satisfacen una necesidad del consumidor y por lo general se consumen de forma regular, en un período de tiempo determinado.

\section{Metodología}

La metodología aplicada fue de tipo exploratoria y documental, la información de tipo secundaria no estadística y dentro de ésta la bibliográfica, la cual se basó en criterios y conceptos emitidos por diferentes autores y expertos sobre temas de aranceles, salvaguardias, seguridad arancelaria, entre otras.

Para los resultados se aplicó la de tipo de exploratoria, documental y secundaria pero estadística, esta última fue extraída en su totalidad de la base de datos del portal estadístico de Corpe Ecuador denominado Trade-Mape, en el cual se registran los datos estadísticos del comercio mundial de todas las partidas arancelarias correspondientes a los productos o bienes con datos 
anuales trimestrales y mensuales de importaciones y exportaciones, en toneladas métricas, en valores monetarios, en este caso en dólares de los Estados Unidos de Norteamérica entre otros.

Los números de las partidas arancelarias que fueron afectadas con la sobretasa arancelaria en el sector de alimentos fue tomado del Servicio Nacional de Aduanas del Ecuador (SENAE), los datos estadísticos comparados corresponden a la tasa arancelaria ad-valoren anterior al incremento con las tasas o valores incrementados, con los datos de esas partidas y tasas, se procedió a la revisión en las cifras del Trade-Mape las importaciones realizadas por el Ecuador en el periodo 2014 al 2016, ya que la medida fue tomada a mediados del año 2014 y la misma tuvo vigencia hasta mayo del 2017, al no existir datos oficiales aún del año 2017, el análisis se realizó del 2014 al 2016; mismo que se realizó sobre el incremento en valores relativos y absolutos, con un análisis de tipo horizontal.

\section{Resultados}

Los resultados de la investigación se presentan a continuación: 


\begin{tabular}{|c|c|c|c|c|c|c|c|c|}
\hline \multirow[b]{4}{*}{ Producto importado } & \multicolumn{8}{|c|}{ Miles de dólares } \\
\hline & \multirow[b]{3}{*}{$\begin{array}{c}\text { Incremento } \\
\%\end{array}$} & \multirow[b]{3}{*}{ valor } & \multirow{3}{*}{$\begin{array}{c}2014 \\
\text { Incremento } \\
\%\end{array}$} & & \multirow[t]{2}{*}{ Años } & \multirow{3}{*}{$\begin{array}{c}2015 \\
\text { Incremento } \\
\%\end{array}$} & \multirow[b]{3}{*}{ Incremento } & \multirow{3}{*}{$\begin{array}{l}2016 \\
\text { valor }\end{array}$} \\
\hline & & & & \multirow[b]{2}{*}{ Incremento } & & & & \\
\hline & & & & & valor & & & \\
\hline $\begin{array}{l}\text { Leche y productos lácteos; huevos de ave; miel natural; } \\
\text { productos comestibles de origen animal, no expresados } \\
\text { ni comprendidos en otra parte }\end{array}$ & $15-25$ & 350 & -24.6 & 67.5 & 264 & 66.3 & 53.7 & 439 \\
\hline Hortalizas, plantas, raíces y tubérculos alimenticios & & 1724 & -17.1 & 344.8 & 1429 & -33.8 & 285.8 & 946 \\
\hline $\begin{array}{l}\text { Frutas y frutos comestibles; cortezas de agrios } \\
\text { (cítricos), melones o sandías }\end{array}$ & 20 & 120106 & -30.5 & 31728.9 & 83447 & -30.3 & 21900.8 & 58179 \\
\hline $\begin{array}{l}\text { Café, incluso tostado o descafeinado; cáscara y } \\
\text { cascarilla de café; sucedáneos del café que contengan } \\
\text { café en cualquier proporción. }\end{array}$ & $15-25$ & 585 & 16.8 & 127.3 & 683 & -28 & 138.9 & 492 \\
\hline Trigo y morcajo (tranquillón & $15-30$ & 1114 & -13.3 & 182.6 & 966 & -17.9 & 169.4 & 793 \\
\hline Harina de trigo o de morcajo (tranquillón & $15-45$ & 17273 & -28.8 & 7750.05 & 12300 & -13.6 & 5502.6 & 10622 \\
\hline $\begin{array}{l}\text { Grasa de cerdo (incluida la manteca de cerdo) y grasa } \\
\text { de ave, excepto las de las partidas } 02.09 \text { ó } 15.03 \text {. }\end{array}$ & 13 & 65 & -46.2 & 8.5 & 35 & 42.9 & 4.6 & 50 \\
\hline $\begin{array}{l}\text { Embutidos y productos similares de carne, despojos o } \\
\text { sangre; preparaciones alimenticias a base de estos } \\
\text { productos }\end{array}$ & 15 & 5219 & -17.4 & 782.85 & 4312 & -1.5 & 646.8 & 4246 \\
\hline $\begin{array}{l}\text { Azúcar de caña o de remolacha y sacarosa } \\
\text { químicamente pura, en estado sólido }\end{array}$ & 25 & 10231 & -24.6 & 2557.8 & 7717 & -43.1 & 1929.3 & 4394 \\
\hline Cacao en grano, entero o partido, crudo o tostado. & $15-25$ & 37360 & -28.1 & 6776.3 & 26854 & -13 & 4835.1 & 23374 \\
\hline
\end{tabular}


Extracto de malta; preparaciones alimenticias de harina, grañones, sémola, almidón, fécula o extracto de malta, que no contengan cacao o con un contenido de

base totalmente desgrasada

Fuente Trade-mape

Elaboración: el autor

Como se aprecia en la tabla 1, en la línea de lácteos el incremento del arancel, fue entre el 15 al 25\%, el valor de las importaciones en el año 2014 , ascendió a 350.000 US\$, los consumidores de esta línea de productos alimenticios tuvieron que pagar un adicional de 67.500 US\$; del año 2014-2015, disminuyó el valor de las importaciones en un 24,6\%, de 350.000 a 264.000 US\$, sin embargo, en el año 2015, los consumidores tuvieron que pagar 53.700 US\$ adicionales; mientras que para el año 2016, al pasar el impacto sicológico, el valor de las importaciones en este concepto se incrementó en el $66,3 \%$ es decir a 439.000 US\$, como consecuencia los ecuatorianos pagaron un valor adicional de 83.450 US\$; en total los valores cancelados por el incremento del arancel en la línea de los alimentos fue de 204.700 US\$. 
En el grupo de las hortalizas, raíces, tubérculos y alimentos, el incremento de los aranceles fue de 20\%, el valor de las importaciones en el año 2014, ascendió a 1.724.000 US\$, los consumidores de esta línea de productos alimenticios tuvieron que pagar un adicional de 344.800 US\$; del año 2014-2015 las importaciones disminuyeron, en un $17.1 \%$, de 1.724.000 US\$ a 1.429.000 US\$, sin embargo, en este año los consumidores tuvieron que pagar 285.800 US\$ adicionales; para el año 2016 el valor de las importaciones continuaron con la tendencia a la baja, a una tasa de $33.8 \%$ de 1.429 .000 US\$ a 946.000 US\$, los ecuatorianos pagaron 189.200 US\$ adicionales; el valor total por efecto del incremento de los aranceles en esta línea de productos alimenticios en estos tres años fue de 819.800 US\$ mismos que asumieron los consumidores.

En la línea de frutos y frutas comestibles, el incremento del arancel fue entre el 20 al 30\%, el valor de las importaciones en esta línea en el año 2014, fue de 120.1millones de US\$, de este valor los consumidores tuvieron que pagar un adicional de 31.7 millones US\$; del año 2014-2015 disminuyó el valor de las importaciones en un 30.5\%, de 120.1 millones de US\$ a 83.4 millones de US\$; sin embargo, en este año los consumidores pagaron 21.9 millones de US\$ adicionales; para el año 2016 el valor de las importaciones continuaron con la tendencia a disminuir con una tasa del 30.3\%, de 83.4 millones de US\$ a 58.2, millones de US\$; los ecuatorianos pagaron 15.3 millones US\$ adicionales; el valor total que se incrementó en esta línea de productos alimenticios fue de 68.9 millones de US.

En el grupo de café, incluso tostado y descafeinado, el incremento de los aranceles fue entre el 15 al 25\%, el valor de las importaciones en el año 2014, fue de 585.000 US\$, de este valor los consumidores de esta línea de productos alimenticios tuvieron que pagar un valor adicional de 127.300 US\$; del año 2014-2015 el valor de las importaciones se incrementó en un 16.8\%, de 585.000 US\$ a 683.000 US\$, como efecto de ello, en el año 2015 los consumidores pagaron 138.900 US\$ adicionales; para el año 2016 el valor de las importaciones se redujo a 492.000 US\$, con una tasa del $28 \%$ de 683.000 US\$ a 492.000, US\$; pese a ello, los ecuatorianos pagaron 108.500 US\$ adicionales; el valor total por efecto del incremento de los aranceles en esta línea de productos alimenticios fue de 374.700 US\$.

En este grupo considerando sólo dos partidas arancelarias, sus valores son altos, el incremento de los aranceles fue entre el 15 el 30\%, el valor de las importaciones en el año 2014, fue de 1.114.000 US\$, sobre este valor los consumidores de esta línea de productos alimenticios tuvieron que pagar un valor adicional de 182.600 US\$, del año 2014-2015 el valor de las importaciones disminuyó un 13.3\%, de 1.114.000 US\$ a 966.000 US\$, sin embargo, en el año 2015 los consumidores pagaron 169.400 US\$ adicionales; para el año 2016 el valor de las importaciones mantuvieron la tendencia a la baja a un valor de 793.000 US\$, con una tasa del $17.9 \%$ de 966.000 US\$ a 793.000, US\$; a pesar de ello, los ecuatorianos pagaron 130.200 dólares US\$ adicionales; el valor total pagado por los consumidores de estos productos alimenticios fue de 509.200 US\$.

Uno de los productos de mayor importación en el Ecuador dentro de la línea alimenticia constituye la harina de trigo que forma parte de este grupo, el porcentaje del incremento de los aranceles en este grupo fue el más alto entre el 15 el 45\%, el valor de las importaciones en el año 2014, fue de 17.3 millones de US\$, sobre este valor los consumidores de esta línea de productos alimenticios tuvieron que pagar un valor adicional de 7.8 millones US\$ por efecto del incremento del arancel; del año 2014-2015, el valor de las importaciones disminuyó en $28.8 \%$, de 17.3 millones de US\$ a 12.3 millones de US\$, a pesar de ello, en el año 2015 los consumidores pagaron 5.5 millones de US\$ adicionales; para el año 2016 el valor de las importaciones mantuvieron la tendencia a la baja con una tasa del $13.6 \%$ de 12.3 millones de US\$ a 10.6 millones de US\$, a pesar de ello, los ecuatorianos pagaron 4.8 millones de US\$ adicionales; el valor total pagado por los consumidores en esta línea de productos alimenticios fue de18.1 millones de US\$. 
Este grupo representa valores menores en las importaciones gravadas con las salvaguardias, el incremento de los aranceles fue del 13\%, el valor de las importaciones en el año 2014, fue de 65.000 US\$, de este valor los consumidores de esta línea de productos alimenticios pagaron un valor adicional de 8.500 US\$ por el incremento del arancel; del año 2014-2015 el valor de las importaciones disminuyeron en un 42.6\%, de 65.000 US\$ a 35.000 US\$, a pesar de ello, en el año 2015 los consumidores pagaron un valor adicional de 4.600 US\$ adicionales; para el año 2016 el valor de las importaciones subió a 50.000 US\$, con una tasa de crecimiento del $42.9 \%$ de 35.000 US\$ a 450.000, US\$; de los cuales, los ecuatorianos tuvimos que pagar 6.500 US\$ adicionales; el valor total por efecto del incremento de los aranceles en esta línea de productos alimenticios es de 19.600 US\$ que asumidos por los ecuatorianos que consumir esos bienes.

Las importaciones de las preparaciones alimenticias de estos productos gravadas con las salvaguardias, tuvieron un incremento del 15\%, el valor de las importaciones en el año 2014, fue de 2.2 millones de US\$, por este valor los consumidores de esta línea de productos alimenticios tuvieron que pagar un valor adicional de 782.850 US\$; del año 2014-2015 el valor de las importaciones se redujo en un $17.4 \%$, de 5.2 millones de US\$ a 4.3 millones de US\$, a pesar de ello, en el año 2015 los consumidores debieron pagar un valor adicional de 646.800 US\$ adicionales; para el año 2016 el valor de las importaciones disminuyó ligeramente a 4.2 millones de US\$, con una tasa de $1.5 \%$ de 4.3 millones de US\$ a 4.2 millones de US\$; a pesar de ello, los consumidores tuvimos que pagar 636.900 US\$ adicionales; el incremento del valor total por efecto del incremento de los aranceles en esta línea de productos alimenticios es de 2.066,6 US\$, asumidos por los consumidores de estos bienes alimenticios.

En esta línea de productos a pesar de constar una sola partida arancelaria, presenta valores muy altos, ésta tuvo un incremento en el orden del 25\%, las importaciones en el año 2014, fueron de 10.2 millones de US\$, sobre este valor los consumidores de esta línea de productos alimenticios pagaron 2.6 millones de US\$ adicionales; del año 2014-2015 el valor de las importaciones se redujeron en un $24.6 \%$, de 10.2 millones de US\$ a 7.7 millones de US\$, sin embargo de esta reducción, en el año 2015 los consumidores debieron pagar un valor adicional de 1.9 millones de US\$; para el año 2016 el valor de las importaciones disminuyó considerablemente con una tasa de $43.1 \%$ de 7.7 millones de US\$ a 4.4 millones de US\$ dólares; pese a ello, los consumidores pagaron 1.1millones de US\$ adicionales; el valor total por efecto del incremento de los aranceles en esta línea de productos alimenticios asciende a 5.6 millones de US\$.

A pesar de que Ecuador es productor y exportador de materia prima para esta línea de productos, las importaciones de productos terminados y derivados tiene un alto valor, así lo demuestra su peso dentro del subsector alimentos importados; las importaciones de esta línea de productos, tuvieron un incremento entre el 15 y el 25\%, las importaciones en el año 2014, fueron de 37.4 millones de US\$, de este valor los consumidores de esta línea de productos alimenticios pagaron un valor adicional de 6.8 millones de US\$; del año 2014-2015, las importaciones se redujeron en un $28.1 \%$, de 37.4 millones de US\$ a 26.9 millones de US\$, sin embargo de esta reducción, en el año 2015 los consumidores debieron pagar un valor adicional de 4.8 millones de US\$; para el año 2016 el valor de las importaciones continuo disminuyendo a 23.4 millones de US\$, con una tasa de $13 \%$ de 26.9 millones de US\$ a 23.4 millones de US\$; sin embargo, los consumidores pagaron 4.2 millones de US\$ adicionales; el valor total por efecto del incremento de los aranceles en esta línea de productos alimenticios asciende a 15.8 millones de US\$.

En este grupo de productos, las importaciones, tuvieron un incremento entre el 15 y el $25 \%$, el valor de las importaciones en el año 2014, ascendieron a 49.9 millones de US\$, de este valor los consumidores de esta línea de productos alimenticios pagaron un valor adicional de 11.6 millones de US\$; del año 2014-2015, las importaciones se redujeron en un $8.3 \%$, de 49.9 millones de US\$ a 45.7 millones de US\$, a pesar de esta reducción, en el año 2015 los consumidores debieron pagar un valor adicional de 10.6 millones de US\$; para el año 2016 las importaciones continuaron con tendencia a la baja disminuyendo a 33.4 millones de US\$, con 
una tasa decreciente de $27 \%$ de 45.7 millones de US\$ a 33.4 millones de US\$; sin embargo de esta reducción, los consumidores pagaron 7.9 millones de US\$ adicionales; sumando todos los valores ocasionados por el incremento de los aranceles en esta línea de productos alimenticios, se tuvo que pagar 30.1 millones de US\$, asumidos por los consumidores de este tipo de bien.

\section{Discusión}

Si se relaciona con la concepción teórica de la aplicación de las salvaguardias sobre los bienes importados, sustentada por diferentes autores y organismos como la Organización Mundial del Comercio (OMC), en la que manifiesta que el objetivo de aplicar la misma será la de proteger a la industria o producción nacional, en el Ecuador, es fácil darse cuenta que este no fue resultado que se esperaba.

Más bien las razones para que el gobierno nacional haya tomado esta decisión de aplicar las salvaguardias a más de 2800 partidas arancelarias de productos importados, dentro de las cuales se encuentran las que contienen productos alimenticios, fueron otras como la de cubrir el gran déficit fiscal ocasionado por la reducción del precio del petróleo y la apreciación del dólar en los países de Colombia y Perú, es decir tenía otro fin, el carácter tributario y de recaudación.

Dentro de la lista de los bienes afectados por esta medida están los de los alimentos ya sea de productos o bienes de consumo final, así como los considerados como materias primas para producir otros bienes de carácter alimenticio, contemplados en los diferentes grupos, como son hortalizas y sus preparaciones, tubérculos y raíces, cereales, carnes y sus preparaciones, frutales. Si bien buena parte de los bienes alimenticios importados no son consumidos por todos los ecuatorianos debido a sus elevados costos frente a los producidos en el país, sin embargo, no es menos cierto que cuando un producto similar, sustituto o complementario sube de precio en los mercados internos por la razón que sea, esto arrastra al resto de productos a la subida de los precios produciéndose un efecto espiral, lo que conlleva a un alza generalizada de los precios; todo esto en un país en donde el nivel de desnutrición a pesar de haber disminuido sigue siendo alto.

Dentro de los principales hallazgos están los siguientes: el valor total que se ha importado en la línea de alimentos, según los registros estadísticos, en los años 2014 al 2016 asciende a 564.6 millones de US\$; de los cuales 243.9, millones de US\$ corresponden al 2014, 183.7 millones de US\$ son del 2015 y 136.9, millones de US\$ corresponden al 2016.

La incidencia que tuvo la medida se ve reflejada claramente en las reducciones de los valores importados en los dos siguientes años de su aplicación, en casi todas las partidas para alimentos, ya que a pesar que subieron los precios de los bienes el valor importado se redujo al disminuir las cantidades importadas, con lo que la oferta de productos alimenticios disminuyó en desmedro de la alimentación de las familias ecuatorianas.

El valor adicional que los ecuatorianos tuvieron que pagar por la incidencia del incremento de las tasas arancelarias en la línea de los alimentos fue de 142.4 millones de US\$, dividido entre los tres años 2014, con 62,5 millones de US\$, 2015 con 46,1 millones de US\$ y 2016 con 34.3 millones de US\$.

La incidencia directa se observó en el impacto económico que sufrieron las familias al tener que pagar un valor adicional para adquirir los alimentos importados, ya que si antes de la medida muchos no los podían adquirir, peor aún con el incremento de los precios por efecto de la subida de los aranceles.

La incidencia final de la fijación de las salvaguardias en el sector alimenticio sin duda está en la disminución de la oferta de alimentos importados, tal como se muestra en las tablas de los distintos grupos de alimentos afectados por las medidas, ya que, en casi en todos los grupos la medida afectó al volumen importado, disminuyendo desde el 20 hasta el 50\% en algunos casos, 
si bien en la teoría estos bienes pueden ser sustituidos por los de producción interna, en un período corto y sin políticas complementarias esto no se puede lograr.

\section{Referencias bibliográficas}

(OMC), A. M. (1994). Información técnica sobre salvaguardia. Obtenido de https://www.wto.org/spanish/tratop_s/safeg_s/safeg_info_s.htm

Hector, M. (2011). Una nota sobre los conceptos de de seguridad alimentaria . Seguridad Alimnetaria Nutricional, conceptos Básicos , 2.

Hoz, A. d. (2013). Generalidades del comercio internacional . Medellin: Esumer Institución universitaria.

J, S. J. (2015). Salvaguardia, mecanismo de defenza a la producción nacional . Mexico: Instituto de investigaciones jurídicas.

Jimenez, R. (2015). Qué son las empresas de bienes de consumo. IZeI.

Juan Gongora, S. M. (2010). La politica arancelaria y el comercio exterior. Comercio exterior, 233.

Maletta, H. (2011). Una nota sobre los concetos de seguridad alimnetaria ,FODEPAL. Seguridad Alimneticia Nutricional, Concetos Básicos , 2.

Manuel, S. P. (2015). Salvaguardia, mecanismo de defenza a la producción nacional. Mexico: Instituto de investigaciones jurídicas.

Parramon, J. M. (2015). Una alternativa al capitalismi neo liberal. Rusia: ECU.

R, R. J. (2015). Qué son las empresas de bienes de consumo. IZeI.

Saldaña, J. (2015). Salvaguadia, mecanismo de defensa a la producción nacional . México: Instituto de investigaciones jurídicas. 